\title{
A Study on Central Lymph Node Metastasis in 543 cNo Papillary Thyroid Carcinoma Patients
}

\author{
Huanhuan Yan, Xiaoqian Zhou, Hui Jin, Xiang Li, Miao Zheng, \\ Xu Ming, Ruitao Wang, and Jun Liu \\ Department of Breast-Thyroid-Vascular Surgery, Shanghai General Hospital of Nanjing Medical University, 201620 Shanghai, China \\ Correspondence should be addressed to Jun Liu; 15300795039@163.com
}

Received 7 January 2016; Revised 9 March 2016; Accepted 10 March 2016

Academic Editor: Giuseppe Damante

Copyright (c) 2016 Huanhuan Yan et al. This is an open access article distributed under the Creative Commons Attribution License, which permits unrestricted use, distribution, and reproduction in any medium, provided the original work is properly cited.

\begin{abstract}
Background. Papillary thyroid carcinoma (PTC) with central lymph node metastases (CLNMs) is common. The objective of this study was to investigate the incidence and risk factors of lymph node metastasis patients with PTC. Patients and Methods. Between January 2013 and February 2015, a retrospective study of 543 patients with PTC undergoing hemithyroidectomy or total thyroidectomy with routine central lymph node dissection (CLND) was analyzed. Clinicopathologic risk factors for CLNM were studied using univariate and multivariate analysis by SPSS 22.0 software. Results. The incidence of CLNMs in PTC patients was $38.1 \%$ (207/543). In the multivariate analysis, male gender $(p<0.001$, OR: 1.984$)$, age $<45$ years $(p<0.001$, OR: 1.934), bilaterality $(p=0.006$, OR: 1.585$)$, tumor size $\geq 0.25 \mathrm{~cm}(p=0.001$, OR: 7.655$)$, and external extension $(p=0.001$, OR: 7.579$)$ were independent risk factors of CLNMs. Furthermore, in PTC patients with tumor size $<0.25 \mathrm{~cm}$, all 7 males and 21 patients with unilaterality were not found to have CLNMs. Conclusions. CLNMs are prevalent in the PTC patients with the following risk factors: male gender, age $<45$ years, bilaterality, tumor size $\geq 0.25 \mathrm{~cm}$, and external extension. PTC patients with tumor size $<0.25 \mathrm{~cm}$, male patients, and patients with unilateral lesion could be considered safe from CLNMs.
\end{abstract}

\section{Introduction}

Papillary thyroid carcinoma (PTC) is the most common form of thyroid gland carcinoma which accounts for $1 \%$ of all human malignant tumors. It was reported that the prognosis of PTC is excellent with a 10-year survival exceeding $90 \%$ [1]. However, approximately $20-50 \%$ of PTC patients were found to have central lymph node metastases (CLNMs) $[2,3]$. There is still a recurrence rate of $8-25 \%$ after primary surgical treatment [4], and many scholars hold the opinions that routine central lymph node dissection (CLND) may decrease the rate of recurrence.

\section{Patients and Methods}

This retrospective study was approved by Department of Breast-Thyroid-Vascular Surgery at Shanghai General Hospital of Nanjing Medical University. From January 2013 to February 2015, a total of 543 PTC patients who underwent radical operation of thyroid were enrolled in this study. Total thyroidectomy and bilateral CLND were considered when the $\mathrm{CN} 0$ patients had bilateral PTC. Total thyroidectomy and ipsilateral CLND might be performed for unilateral PTC patients who met one or more of the following conditions according to the NCCN guidelines: radiation history, known distance metastases, extrathyroidal extension, and tumor size $>4 \mathrm{~cm}$ in diameter. Other unilateral PTC patients underwent unilateral and contralateral partial resection of thyroid gland or hemithyroidectomy with ipsilateral CLND. The CLN was group VI lymph nodes. Its upper bound was to the thyroid cartilage, and its lower bound was to thymus, and both sides were to the inner side of the central bilateral carotid sheath, including lymph nodes prior to cricothyroid membrane, lymph nodes surrounding thyroid, and paratracheal and prelaryngeal lymph node. Intraoperative frozen section (FS) is a popular method for doctors to determine surgical extent in surgery. It was reported that the accuracy rates for FS in thyroid surgery was $97.8 \%$ [5]. And intraoperative FS was 
performed in all patients. Preoperative ultrasonography, preoperative thyroid function tests, and postoperative pathologic examination were performed in all patients.

Based on the NCCN guidelines for PTC [6], all of the 543 patients with clinically lymph node that was negative according to the preoperative physical examination and ultrasound underwent the surgical treatment. Total thyroidectomy with bilateral/unilateral CLND was performed in 419 patients. 79 patients underwent unilateral and contralateral partial resection of thyroid gland with bilateral/unilateral CLND. Hemithyroidectomy with bilateral/unilateral CLND was performed in 39 patients. Six patients performed secondary surgical intervention because they were diagnosed as having benign lesions according to intraoperative FS, but the postoperative pathological diagnosis of the first surgery was PTC. In this study, the preoperative fine needle aspiration was carried out on 337 patients, and 322 cases were diagnosed with PTC. From a total of 543 patients, 537 cases were diagnosed with PTC by the intraoperative FS, and 6 cases were diagnosed postoperatively after the histopathological examination of thyroid gland. LT4 was given to all the patients as an alternative treatment besides the inhibition of TSH therapy.

\section{Statistics Analysis}

Chi-square test or Fisher's exact test was used to analyze the significance of differences of the categorical variables and logistic regression was performed to analyze the multiplicity. All the data were processed using Statistic Package for Social Science (SPSS 22.0) and $p<0.05$ was considered as a statistically significant difference.

\section{Results}

4.1. Clinical and Pathological Characteristics. 410 (75.5\%) females and 133 (24.5\%) males were included in this study. The average age of all the patients was 47 years, ranging from 16 to 78 years. The mean size of the tumors was $0.95 \pm 0.73 \mathrm{~cm}$ (ranging from $0.1 \mathrm{~cm}$ to $5 \mathrm{~cm}$ ). $132(24.3 \%$ ) patients had bilateral lesions, 180 (33.1\%) cases had multifocal lesions, 517 (95.2\%) cases had tumor $\geq 0.25 \mathrm{~cm}$ in size, and $36(6.6 \%)$ cases had PTC with extrathyroidal extension. 190 (35.0\%) cases had PTC with Hashimoto's thyroiditis (HT) and 78 (14.4\%) cases had abnormal thyroid function.

4.2. Risk Factors for PTC with CLNMs. We analyzed these factors including the gender, age, bilaterality, multifocality, tumor size, extrathyroidal extension, HT, and abnormal thyroid function to find the risk factors for PTC patients with CLNMs. In the univariate analysis, male gender $(p=0.012)$, age $<45$ years $(p<0.001)$, bilateral lesions $(p=0.042)$, tumor size $\geq 0.25 \mathrm{~cm}(p=0.003)$, and external invasion $(p=0.003)$ were significantly associated with CLNMs. Multifocal lesions, HT, and abnormal thyroid function were not significant for CLNMs (Table 1).

The multivariate analysis (Table 2 ) showed that the male gender $(p<0.001$, OR: 1.984$)$, age $<45$ years $(p<0.001$, OR: 1.934), bilateral lesions ( $p=0.006$, OR: 1.585$)$, tumor size $\geq 0.25 \mathrm{~cm}(p=0.001$, OR: 7.579$)$, and external invasion
TABLE 1: Univariate analysis for PTC patients with CLND.

\begin{tabular}{|c|c|c|c|c|}
\hline \multirow{2}{*}{ Risk factors } & \multicolumn{2}{|c|}{ CLN } & \multicolumn{2}{|c|}{ Univariate analysis } \\
\hline & Positive & Negative & $\chi^{2}$ & $p$ \\
\hline Gender & & & 6.385 & 0.012 \\
\hline Female & 144 & 266 & & \\
\hline Male & 63 & 70 & & \\
\hline Age (years) & & & 11.2 & 0.001 \\
\hline$<45$ & 102 & 117 & & \\
\hline$\geq 45$ & 105 & 335 & & \\
\hline Bilaterality & & & 4.131 & 0.042 \\
\hline Yes & 61 & 73 & & \\
\hline No & 146 & 263 & & \\
\hline Multifocality & & & 3.797 & 0.051 \\
\hline Yes & 79 & 101 & & \\
\hline No & 128 & 235 & & \\
\hline Tumor size $(\mathrm{cm})$ & & & 9.407 & 0.002 \\
\hline$<0.25$ & 2 & 24 & & \\
\hline$\geq 0.25$ & 205 & 312 & & \\
\hline Extrathyroidal extension & & & 8.639 & 0.003 \\
\hline Yes & 22 & 14 & & \\
\hline No & 185 & 322 & & \\
\hline HT & & & 2.44 & 0.118 \\
\hline Yes & 64 & 126 & & \\
\hline No & 143 & 210 & & \\
\hline Abnormal thyroid function & & & 0.102 & 0.75 \\
\hline Yes & 31 & 47 & & \\
\hline No & 176 & 289 & & \\
\hline
\end{tabular}

$(p=0.002$, OR: 2.370) were independent risk factors for CLNMs in PTC patients.

We grouped all the PTC patients into five groups based on the size of tumors: $<0.25 \mathrm{~cm}, \geq 0.25$ and $<0.5 \mathrm{~cm}, \geq 0.5$ and $<0.75 \mathrm{~cm}, \geq 0.75$ and $<1 \mathrm{~cm}, \geq 1 \mathrm{~cm}$. And the rate of CLNMs increased as the tumor size increased. There were significant differences for these groups $(p<0.001)$ (Table 3).

Among the 543 cN0 PTC patients, 38.1\% (207/543) were found to pathologically have CLNMs. Table 4 showed that no CLNMs were found in all 7 males and 21 patients with unilateral lesion in cN0 PTC patients with tumor size $<0.25 \mathrm{~cm}$. The percentages of patients with CLNMs whose conditions met only zero, one, two, three, four, or all of the five risk factors were 0 (0/6), 22.5\% (39/173), 43.7\% (104/238), 46.3\% (50/108), $77.8 \%$ (14/18), and 0. Among the 6 patients without these five risk factors, $0 \%(0 / 6)$ were found to have CLNMs (Table 4).

\section{Discussion}

Papillary thyroid carcinoma which is considered to have relatively good prognosis still has at least $10 \%$ risk of recurrence in long-term follow-up [7-9]. CLNMs are the most important variable known to increase the risk of local recurrence [10]. A large study found that the mortality of PTC patients with 
TABLE 2: Multivariate analysis for risk factors of central lymph node metastasis.

\begin{tabular}{|c|c|c|c|c|c|c|}
\hline Variables & SE & Wald & $p$ value & $\operatorname{Exp}(b)$ & \multicolumn{2}{|c|}{ 95\% CI of $\operatorname{Exp}(b)$} \\
\hline Gender (male versus female) & 0.169 & 16.544 & 0.000 & 1.984 & 1.427 & 2.762 \\
\hline Age ( $<45$ versus 45 years $)$ & 0.165 & 15.864 & 0.000 & 1.934 & 1.397 & 2.674 \\
\hline Bilaterality (yes versus no) & 0.167 & 7.655 & 0.006 & 1.585 & 1.144 & 2.198 \\
\hline Tumor size $(<0.25$ versus $\geq 0.25 \mathrm{~cm})$ & 0.608 & 11.11 & 0.001 & 7.579 & 2.303 & 24.938 \\
\hline Extrathyroidal extension (yes versus no) & 0.278 & 9.668 & 0.002 & 2.370 & 1.376 & 4.082 \\
\hline Constant & 1.430 & 0.027 & 0.101 & 0.057 & & \\
\hline
\end{tabular}

TABLE 3: Relationship between tumor diameter and CLNMs.

\begin{tabular}{lcccc}
\hline \multirow{2}{*}{ Tumor size $(\mathrm{cm})$} & \multicolumn{2}{c}{ CLN } & \multicolumn{2}{c}{ Univariate analysis } \\
& Positive & Negative & $\chi^{2}$ & $p$ \\
\hline$<0.25$ & 2 & 24 & 48.674 & $<0.001$ \\
$0.25-0.49$ & 22 & 75 & & \\
$0.50-0.74$ & 48 & 111 & & \\
$0.75-0.99$ & 25 & 30 & & \\
$\geq 1$ & 110 & 96 & & \\
\hline
\end{tabular}

CLNMs was much higher than that of patients without CLNMs [11]. Pellegriti et al. [12] kept the option that the development of distant metastases was associated with the presence of lymph node metastases at presentation. The role of routine CLND for $\mathrm{CN} 0$ PTC remains controversial. More and more scholars recommend that CLND is necessary for PTC patients because of the greater rate of CLNMs. Wang et al. [10] reported that $44.1 \% \mathrm{cN} 0 \mathrm{PTC}$ patients were found to have CLNMs. Jiang et al. [13] reported that nearly $53.71 \%$ cN0 PTC patients had CLNMs. However, Machens et al. [14] considered that CLNMs were associated with local recurrence and distant metastasis but did not impair survival. Therefore, it is essential to investigate the indications for cN0 PTC patients.

Obviously, male gender, age $<45$ years old, bilaterality, tumor size $\geq 0.25 \mathrm{~cm}$, and external invasion were independent risk factors for cN0 PTC patients. Several studies demonstrated that male PTC patients have higher significant risk of CLNMs [15, 16]. However, Jiang et al. [13] found that the gender had no association with CLNMs. In our study, the proportion of the male gender with CLNMs was significantly higher than that of female gender $(47.4 \%$ versus $35.1 \%, p<$ 0.001, OR: 1.984). Age is often used to judge the stage of the differentiated thyroid carcinoma. We found that younger PTC patients $(<45$ years old $)$ were at higher risk of occurring CLNMs (46.6\% versus $23.9 \%, p<0.001$, OR: 1.934). Ahn et al. [17] analyzed $916 \mathrm{cN0}$ PTC patients and had similar finding that the rate of CLNMs was considerably higher in the cases of younger patients ( $p<0.001$; OR: 2.357). Jiang et al. [13] believed that age $\geq 35$ years was a good prognostic factor for PTC patients with CLNMs. In agreement with Pellegriti et al. [12] and Vasileiadis et al. [18], we found that bilateral lesions made a meaningful difference with a higher percentage to develop CLNMs from the unilaterality (45.5\% versus $35.7 \%, p=0.006$, OR: 1.585$)$. It was accepted that the tumor size is associated with lymph node metastasis. Chang et al. [15] and Zhang et al. [19] have demonstrated that larger tumor size $(>0.5 \mathrm{~cm},>0.6 \mathrm{~cm})$ was associated with CLNMs. In our study, PTC patients with tumor size $\geq 0.25 \mathrm{~cm}$ were more prone to develop CLNMs (39.7\% versus 7.7\%, $p=0.001$, OR: 7.579). Many studies have demonstrated that external invasion is a risk factor of CLNMs for PTC patients $[4,13$, $15,18]$. Our study showed that nearly $61.1 \%$ percent of PTC patients with external invasion was found to have CLNMs and the multivariate analysis indicated that external invasion was an independent risk factor for CLNMs (61.1\% versus $36.5 \%, p=0.002$, OR: 2.370 ).

With the development of ultrasound, palpation, fine needle aspiration (FNA), and intraoperative frozen section, increasing small malignant thyroid nodules were checked out. Before the dispute regarding $[20,21]$ the operation for PTC patients with tumor size $\leq 1 \mathrm{~cm}$, studies shall need to advance. In this study, the rate of tumor size with CLNMs increased, obviously, with the increase of tumor size in a certain range $(<0.25 \mathrm{~cm}: 7.6 \%, \geq 0.25$ and $<0.5 \mathrm{~cm}: 22.7 \%$, $\geq 0.5$ and $<0.75 \mathrm{~cm}: 30.1 \%, \geq 0.75$ and $<1 \mathrm{~cm}: 45.5 \%, \geq 1 \mathrm{~cm}$ : $53.4 \%$ ). $7.6 \%$ of PTC patients with tumor size $<0.25 \mathrm{~cm}$ were confirmed to have metastases in central lymph node, while about $39.7 \%$ of PTC patients whose tumor size was $\geq 0.25 \mathrm{~cm}$ were confirmed to have CLNMs. The incidence of CLNMs in PTC patients with tumor size $\geq 0.25$ and $<0.5 \mathrm{~cm}$ was $22.7 \%$. And the incidence of CLNMs in PTC patients with tumor size $<0.5 \mathrm{~cm}$ was reported to be $20.6-26.2 \%[13,15,17]$. Therefore, we think that CLND should be performed in PTC patients with tumor size $\geq 0.25 \mathrm{~cm}$.

In this study, the incidence of CLNMs in PTC patients was $38.1 \%$. Similar studies in cN0 PTC patients reported that the incidence of CLNMs was $35.0-43.6 \%$ [15, 16, 22]. The clinic risk factors for PTC patients with CLNMs were analyzed above, and we also evaluated the incidence of CLNMs with every conceivable combination to further analyze the indication of CLND. Eliminating tumor size $\geq 0.25 \mathrm{~cm}$, no CLNMs were found in male patients and patients with unilateral lesion. In the present studies, CLND is necessary to be performed in the PTC patients because CLNMs are associated with a higher risk of recurrence [23, 24]. Hence, CLND should be performed in the patients with risk factors, and we recommend that, in cN0 PTC patients with tumor size $<0.25 \mathrm{~cm}$, male patients and patients with unilateral lesion should be considered safe.

In this study, some limitations should be considered. First of all, this retrospective study was short of the prognosis information. Secondly, all patients are required to have longterm follow-up to analyze the significance of CLND for cN0 PTC patients. 
TABLE 4: Risk factors in PTC patients with tumor size $<0.25 \mathrm{~cm}$ and $\geq 0.25 \mathrm{~cm}$.

\begin{tabular}{|c|c|c|c|c|}
\hline \multirow{3}{*}{ Risk factors } & \multicolumn{2}{|c|}{ Tumor size $<0.25 \mathrm{~cm}(26)$} & \multicolumn{2}{|c|}{ Tumor size $\geq 0.25 \mathrm{~cm}$} \\
\hline & \multicolumn{4}{|c|}{ CLN } \\
\hline & Positive & Negative & Positive & Negative \\
\hline \multicolumn{5}{|l|}{ Gender } \\
\hline Female & 2 & 17 & 142 & 249 \\
\hline Male & 0 & 7 & 63 & 63 \\
\hline \multicolumn{5}{|l|}{ Age (years) } \\
\hline$<45$ & 1 & 9 & 101 & 108 \\
\hline$\geq 45$ & 1 & 15 & 104 & 204 \\
\hline \multicolumn{5}{|l|}{ Bilaterality } \\
\hline Yes & 2 & 3 & 59 & 70 \\
\hline No & 0 & 21 & 146 & 242 \\
\hline \multicolumn{5}{|c|}{ Extrathyroidal extension } \\
\hline Yes & 0 & 0 & 22 & 14 \\
\hline No & 2 & 24 & 183 & 298 \\
\hline
\end{tabular}

\section{Conclusions}

In summary, the incidence of PTC patients with CLNMs is $38.1 \%$ in our study. Male gender, age $<45$ years, bilaterality, tumor size $\geq 0.25 \mathrm{~cm}$, and external extension were considered as independent risk factors for PTC patients. On this account, we think precautionary CLND should be considered for $\mathrm{cN} 0$ PTC patients with risk factors. And we believe that, in cN0 PTC patients with tumor size $<0.25 \mathrm{~cm}$, male patients and patients with unilateral lesion could avoid CLND.

\section{Competing Interests}

The authors declare that they have no competing interests.

\section{References}

[1] R. R. Malterling, R. E. Andersson, S. Falkmer, U. Falkmer, E. Niléhn, and J. Jrhult, "Differentiated thyroid cancer in a Swedish county-long-term results and quality of life," Acta Oncologica, vol. 49, no. 4, pp. 454-459, 2010.

[2] American Thyroid Association Surgery Working Group, American Association of Endocrine Surgeons, American Academy of Otolaryngology-Head and Neck Surgery et al., "Consensus statement on the terminology and classification of central neck dissection for thyroid cancer," Thyroid, vol. 19, no. 11, pp. 1153$1158,2009$.

[3] T.-A. Moo, J. McGill, J. Allendorf, J. Lee, T. Fahey III, and R. Zarnegar, "Impact of prophylactic central neck lymph node dissection on early recurrence in papillary thyroid carcinoma," World Journal of Surgery, vol. 34, no. 6, pp. 1187-1191, 2010.

[4] K. Guo and Z. Wang, "Risk factors influencing the recurrence of papillary thyroid carcinoma: a systematic review and metaanalysis," International Journal of Clinical and Experimental Pathology, vol. 7, no. 9, pp. 5393-5403, 2014.

[5] B. Cetin, S. Aslan, C. Hatiboglu et al., "Frozen section in thyroid surgery: is it a necessity?" Canadian Journal of Surgery, vol. 47, no. 1, pp. 29-33, 2004.
[6] NCCN Clinical Practice Guidelines in Oncology (NCCN Guidelines), Thyroid Carcinoma, Version 2, 2014, http://www .nccn.org.

[7] S. I. Sherman, P. Angelos, D. W. Ball et al., "Thyroid carcinoma," Journal of the National Comprehensive Cancer Network, vol. 3, pp. 404-457, 2005.

[8] N. A. Samaan, P. N. Schultz, R. C. Hickey et al., "The results of various modalities of treatment of well differentiated thyroid carcinomas: a retrospective review of 1599 patients," Journal of Clinical Endocrinology and Metabolism, vol. 75, no. 3, pp. 714720, 1992.

[9] S. A. Hundahl, B. Cady, M. P. Cunningham et al., "Initial results from a prospective cohort study of 5583 cases of thyroid carcinoma treated in the United States during 1996. U.S. and German Thyroid Cancer Study Group. An American College of Surgeons Commission on Cancer Patient Care Evaluation study," Cancer, vol. 89, pp. 202-217, 1996.

[10] Q. Wang, B. Chu, J. Zhu et al., "Clinical analysis of prophylactic central neck dissection for papillary thyroid carcinoma," Clinical and Translational Oncology, vol. 16, no. 1, pp. 44-48, 2014.

[11] Y. D. Podnos, D. Smith, L. D. Wagman, and J. D. I. Ellenhorn, "The implication of lymph node metastasis on survival in patients with well-differentiated thyroid cancer," American Surgeon, vol. 71, no. 9, pp. 731-734, 2005.

[12] G. Pellegriti, C. Scollo, G. Lumera, C. Regalbuto, R. Vigneri, and A. Belfiore, "Clinical behavior and outcome of papillary thyroid cancers smaller than $1.5 \mathrm{~cm}$ in diameter: study of 299 cases," Journal of Clinical Endocrinology and Metabolism, vol. 89, no. 8, pp. 3713-3720, 2004.

[13] L.-H. Jiang, C. Chen, Z. Tan et al., "Clinical characteristics related to central lymph node metastasis in cN0 papillary thyroid carcinoma: a retrospective study of 916 patients," International Journal of Endocrinology, vol. 2014, Article ID 385787, 6 pages, 2014.

[14] A. Machens, R. Hinze, O. Thomusch, and H. Dralle, "Pattern of nodal metastasis for primary and reoperative thyroid cancer," World Journal of Surgery, vol. 26, no. 1, pp. 22-28, 2002.

[15] Y. W. Chang, H. S. Kim, H. Y. Kim, J. B. Lee, J. W. Bae, and G. S. Son, "Should central lymph node dissection be considered for 
all papillary thyroid microcarcinoma?" Asian Journal of Surgery, 2015.

[16] A. M. Thompson, M. Robin, A. Hayen et al., "A preoperative nomogram for the prediction of ipsilateral central compartment lymph node metastases in papillary thyroid cancer," Thyroid, vol. 24, no. 4, pp. 675-682, 2014.

[17] B. H. Ahn, J. R. Kim, H. C. Jeong, J. S. Lee, E. S. Chang, and Y. H. Kim, "Predictive factors of central lymph node metastasis in papillary thyroid carcinoma," Annals of Surgical Treatment and Research, vol. 88, no. 2, pp. 63-68, 2015.

[18] I. Vasileiadis, E. Karakostas, G. Charitoudis et al., "Papillary thyroid microcarcinoma: clinicopathological characteristics and implications for treatment in 276 patients," European Journal of Clinical Investigation, vol. 42, no. 6, pp. 657-664, 2012.

[19] L. Zhang, W.-J. Wei, Q.-H. Ji et al., "Risk factors for neck nodal metastasis in papillary thyroid microcarcinoma: a study of 1066 patients," Journal of Clinical Endocrinology and Metabolism, vol. 97, no. 4, pp. 1250-1257, 2012.

[20] G. Conzo, P. G. Calò, A. A. Sinisi et al., "Impact of prophylactic central compartment neck dissection on locoregional recurrence of differentiated thyroid cancer in clinically nodenegative patients: a retrospective study of a large clinical series," Surgery, vol. 155, no. 6, pp. 998-1005, 2014.

[21] G. Conzo, C. Mauriello, G. Docimo et al., "Clinicopathological pattern of lymph node recurrence of papillary thyroid cancer. Implications for surgery," International Journal of Surgery, vol. 12, supplement 1, pp. S194-S197, 2014.

[22] W. Wang, J. Gu, J. Shang, and K. Wang, "Correlation analysis on central lymph node metastasis in 276 patients with cN0 papillary thyroid carcinoma," International Journal of Clinical and Experimental Pathology, vol. 6, no. 3, pp. 510-515, 2013.

[23] S.-M. Chow, S. C. K. Law, J. K. C. Chan, S.-K. Au, S. Yau, and W.H. Lau, "Papillary microcarcinoma of the thyroid-prognostic significance of lymph node metastasis and multifocality," Cancer, vol. 98, no. 1, pp. 31-40, 2003.

[24] D. Simon, P. E. Goretzki, J. Witte, and H. D. Röher, "Incidence of regional recurrence guiding radicality in differentiated thyroid carcinoma," World Journal of Surgery, vol. 20, no. 7, pp. 860-866, 1996. 


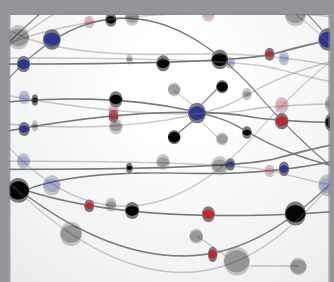

The Scientific World Journal
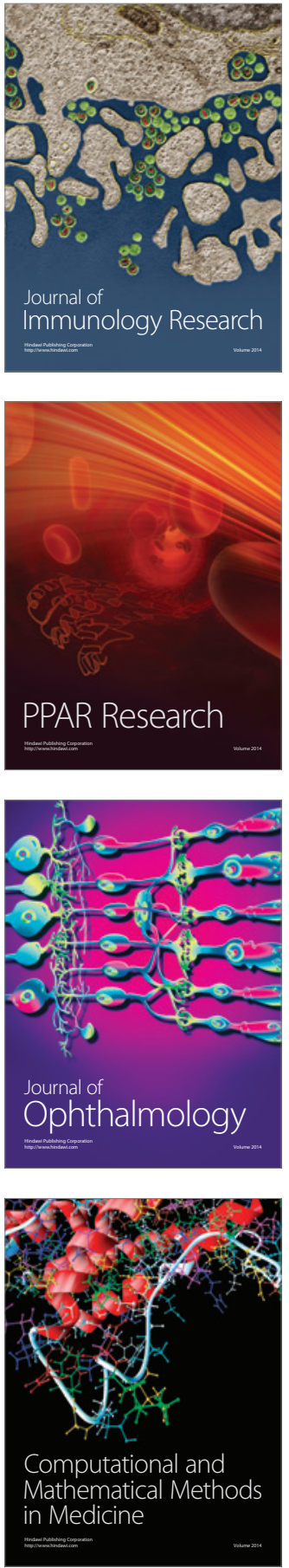

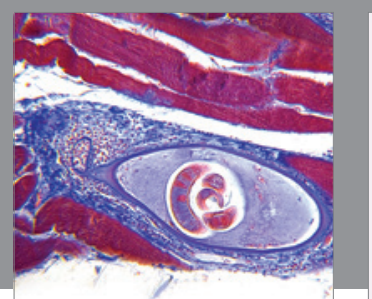

Gastroenterology Research and Practice

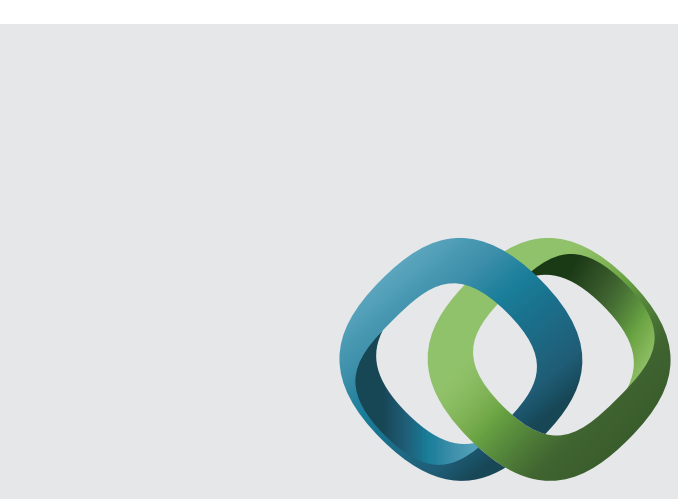

\section{Hindawi}

Submit your manuscripts at

http://www.hindawi.com
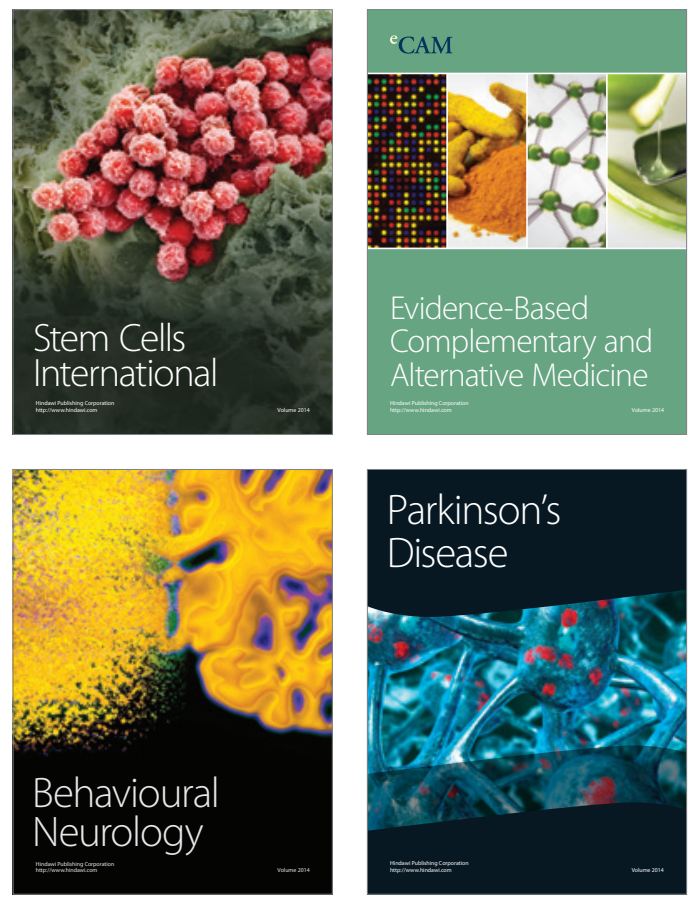
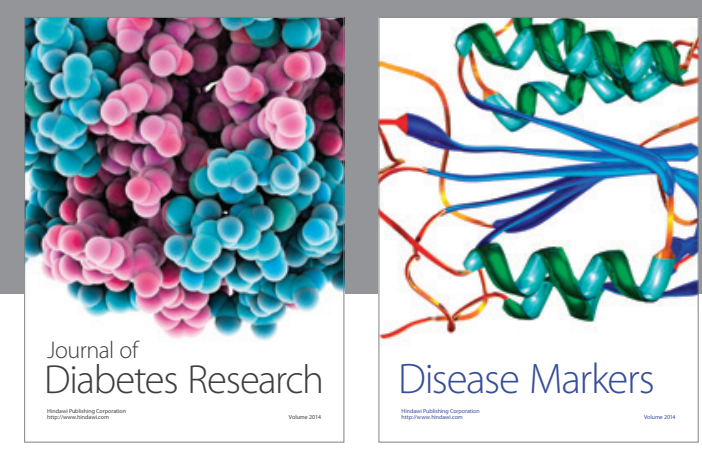

Disease Markers
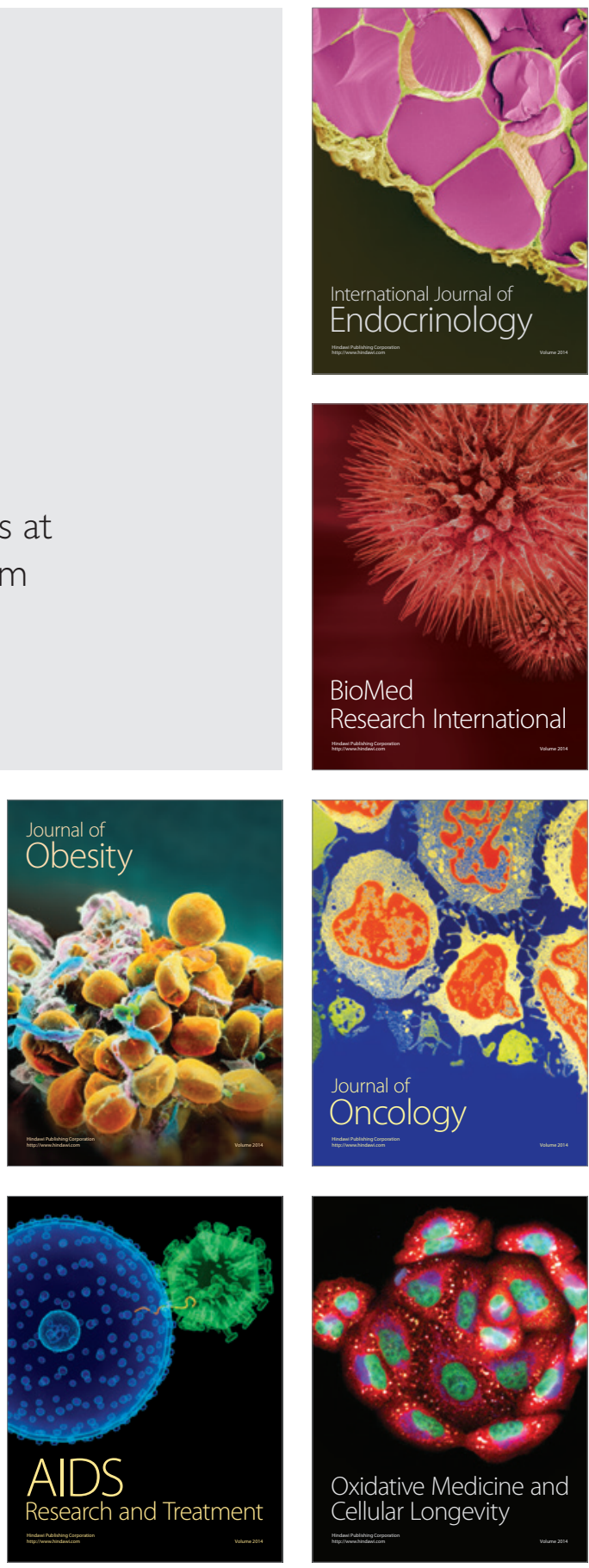